\title{
Synchronized Dynamics and Nonequilibrium Steady States in a Stochastic Yeast Cell-Cycle Network
}

\author{
Hao Ge* Hong Qian ${ }^{\dagger} \quad$ Min Qian ${ }^{\ddagger}$
}

October 26, 2018

\begin{abstract}
Applying the mathematical circulation theory of Markov chains, we investigate the synchronized stochastic dynamics of a discrete network model of yeast cell-cycle regulation where stochasticity has been kept rather than being averaged out. By comparing the network dynamics of the stochastic model with its corresponding deterministic network counterpart, we show that the synchronized dynamics can be soundly characterized by a dominant circulation in the stochastic model, which is the natural generalization of the deterministic limit cycle in the deterministic system. Moreover, the period of the main peak in the power spectrum, which is in common use to characterize the synchronized dynamics, perfectly corresponds to the number of states in the main cycle with dominant circulation. Such a large separation in the magnitude of the circulations, between a dominant, main cycle and the rest, gives rise to the stochastic synchronization phenomenon.
\end{abstract}

KEY WORDS: Boltzmann machine; circulation theory; Hopfield network; nonequilibrium steady state; synchronization; yeast cell cycle

\section{Introduction}

Synchronization is an important characteristics of many biological networks [32, 35] whose dynamics has been modelled traditionally by deterministic, coupled nonlinear ordinary differential equations in terms of regulatory mechanisms and kinetic parameters [20, 4]. Two important classes of biological networks which have attracted wide attentions in recent

${ }^{*}$ School of Mathematical Sciences, Peking University, Beijing 100871, P.R.C.; email: edmund_ge@tom.com

${ }^{\dagger}$ Department of Applied Mathematics, University of Washington, Seattle, Washington 98195, U.S.A.; email: qian@amath.washington.edu

${ }^{\ddagger}$ School of Mathematical Sciences, Peking University, Beijing 100871, P.R.C. 
years are neural networks [31 and cellular biochemical networks [8]. A deterministic model, however, only describes the averaged behavior of a system based on large populations; it can not capture the temporal fluctuations of a small biological system with either extrinsic or intrinsic noise. For example, neuronal firing has an inherent variability, and many biochemical regulatory networks inside a cell consist of molecular species with low concentrations, where stochastic models with chemical master equations (CME) based on biochemical reaction stoichiometry, molecular numbers, and kinetic rate constants should be developed. Such an approach has already provided important insights and quantitative characterizations of a wide range of biochemical systems [19, 33, 5, 6, 24, 37]. We refer to 34] for a good introduction to stochastic modelling in biology.

As there is a growing awareness and interest in studying the effects of noise in biological networks, it becomes more and more important to quantitatively characterize the synchronized dynamics mathematically in stochastic models, because the concepts of limit cycle and fixed phase difference no longer holds in this case. Instead, physicists and biologists always have to characterize synchronized dynamics by the distinct peak of its power spectrum or just only by observing the stochastic trajectories, which however may cause ambiguities in the conclusion. Therefore, a logical generalization of limit cycle in stochastic models needs to be developed.

On the other hand, from the view of statistical physics, these stochastic models for systems cell biology exhibit nonequilibrium steady states (NESS) in which nonequilibrium cycle fluxes necessarily emerge [22]. The relation between an NESS and traditional nonlinear dynamics, in particular bistability [25] and limit-cycle oscillations, has been discussed recently [23, 24].

We have recently developed a rather complete mathematical theory of nonequilibrium steady state (NESS, 14]) of open systems, taking Markov chains as the model of biochemical systems. One of the most important concepts in the mathematical theory of NESS is the circulation(also called cycle fluxes) [14, which corresponds to the cycle kinetics in open chemical systems [10, 22]. Actually, since the stochastic circulation in NESS is defined as the time-averaged frequency of each cycle in the sense of trajectory, it can also be regarded as a quantitative characterization for the intensities of stochastic synchronized dynamics in different attractor basins. This paper will thoroughly investigate the interplay described above in a currently interested stochastic cell-cycle network model.

Cell cycle is one of important biological processes whose underlying molecular networks have been extensively studied. Tyson and his coworkers have studied mathematical models for yeast cell cycle based on differential equations [2]. Their key finding is that cell cycle is a hysteresis loop, via saddle-node bifurcations, driven by the periodic changes in cell mass due to cell growth and division [4]. The biological "check points" correspond to the 
steady states of the dynamical system [3].

However, in many cellular biochemical modelling, a detailed, molecular number-based CME model is not warranted because of a lack of quantitative experimental data. Thus alternatively, one may try to develop discrete state network, such as Hopfield network and Boltzmann machine, model of a complex biological system using the available information on the activation and repression from one signaling molecules to another, e.g., the kind of signaling wiring diagram like Fig. 1,

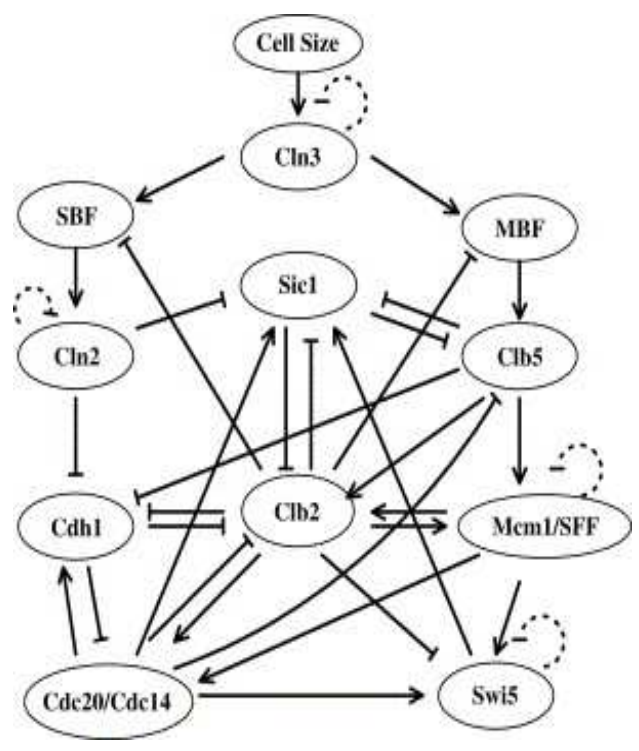

Figure 1: The cell-cycle network of the budding yeast. Each node represents a protein or a protein complex. Arrows are positive regulation, T-lines are negative regulation, dotted T-loops are degradation. It is just Fig. 1 from [38] with permission.

Recently, Li, et.al. [18 have developed a discrete deterministic Boolean model by applying the approach of Hopfield for neural networks to the yeast cell-cycle regulatory network with 11 nodes according to Fig. 1. They have found that the topology of the network provides significant robustness of the dynamics toward the check points, i.e., steady states. The deterministic Boolean model has been further extended to incorporate stochastic dynamics [38]. Several biologically interesting results have been obtained; these include the numerical evidence for the existence of a single dominant cell cycle and its robustness under a large range of noise level.

In the present paper, we will investigate the synchronized stochastic dynamics of the discrete network model of yeast cell-cycle regulation where stochasticity has been kept rather than being averaged out from the start, applying the mathematical circulation theory of Markov chains. By comparing the network dynamics of the stochastic model with its corresponding deterministic network counterpart, we show that the synchronized dynamics can be soundly characterized by a dominant circulation in the stochastic model, 
which is the natural generalization of the deterministic limit cycle in the deterministic system.

It is shown that a large separation in the magnitude of the circulations, between a dominant, main cycle and the rest, gives rise to the stochastic synchronization phenomenon, and the power spectrum of the trajectory has a main peak, whose period converges perfectly to the number of states in the dominant cycle. Furthermore, the net circulation of the dominant cycle increases monotonically with the noise-strength parameter $\beta$, approaching to its deterministic limit. Together, these observations provide a clear picture of the nature of the synchronization in a stochastic network in terms of the probabilistic circulation of NESS. The main part of the present paper, Section IV presents our study on the synchronization and dominant circulation in the cell-cycle network through numerical computations.

For the completeness of the work, we first give a theoretical sketch of some relevant results on biological networks in Section II, including a classification of the deterministic and stochastic Boolean networks and their correspondence. Then a short review of the mathematical theory of stochastic circulation for Markov chains is introduced and applied to Hopfield network and Boltzmann machine in Section III. It is shown that the stochastic Boolean network is reversible if and only if the matrix $T$ in the model is symmetric, and the net NESS circulation is strictly positive as long as the probability of the directed cycle is larger than that of its reversal. Finally, the conclusion and some further discussions are provided in Section V.

\section{Theoretical Sketch of Some Relevant Results on Biolog- ical Networks: Hopfield networks, Boltzmann machines, and Their Relations}

We first give a brief account of the deterministic Hopfield networks and its stochastic incarnation, the Boltzmann machines. Since the influential work of J.J. Hopfield in 1980s' [11, 12], the deterministic Boolean (Hopfield) network has been applied to various fields of sciences. Amit [1] has introduced a temperature-like parameter $\beta$ that characterizes the noise in the network and constructed a probabilistic Boolean network called Boltzmann machine. The Hopfield networks and Bolzmann machines have found wide range of applications in biology. They can be mainly categorized into several classes as follows [1].

Let us suppose $N$ is a fixed integer, $S=\{1,2, \cdots, N\}$. We take the state space as $\{-1,1\}^{S}$.

\section{Model A: deterministic}

A1 (discrete time, synchronous, McCulloch-Pitts): Denote the state of the $n$th step as 
$X_{n}=\left(X_{n}^{1}, X_{n}^{2}, \cdots, X_{n}^{N}\right)$, then the dynamic is

$$
X_{n+1}^{i}=\operatorname{sign} 1\left(\sum_{j=1}^{N} T_{i j} X_{n}^{j}-U_{i}\right), \text { if } \sum_{j=1}^{N} T_{i j} X_{n}^{j} \neq U_{i}
$$

and if $\sum_{j=1}^{N} T_{i j} X_{n}^{j}=U_{i}$, then $X_{n+1}^{i}$ randomly choose 1 or -1 with probability $\frac{1}{2}$ respectively. $U_{i}(1 \leq i \leq N)$, given a priori, are called the threshold of the $i$ th unit.

A2 (continuous time, synchronous): Every state has an exponentially distributed stochastic waiting-time, with mean waiting-time $\lambda^{-1}$, then chooses the next state by the same rule of model $A 1$.

A3 (discrete time, asynchronous, Hopfield): The neurons are updated one by one, in some prescribed sequence, or in a random order. If the previous state $\sigma$ satisfies $\sum_{j=1}^{N} T_{i j} \sigma_{j}>U_{i}$, then $\sigma_{i}$ changes to be 1 , otherwise changes to be -1 .

A4 (continuous time, asynchronous): Every state has an exponentially distributed stochastic waiting-time, with rate constant $\lambda$, then chooses the next state by the same rule of model $A 3$.

Remark II.1 The $(-1,1)$ representation and the $(0,1)$ representation are equivalent. Biologists usually use the $(0,1)$ representation in modelling biological networks, where 0 and 1 denote the resting and activated states, respectively. Physicists usually use the $(-1,1)$ representation for spin systems.

Remark II.2 Note that by deterministic, we mean the transition from one state to the next is deterministic. But the systems with continuous-time will still behave stochastically due to the Poisson nature in the transition time.

\section{Model B: stochastic Boltzmann machines}

B1 (discrete time, synchronous): Consider the Markov chain

$$
\left\{X_{n}=\left(X_{n}^{1}, X_{n}^{2}, \cdots, X_{n}^{N}\right), n=0,1,2, \cdots\right\}
$$

on state space $\{-1,1\}^{S}$, with transition probability given as follows: for each pair of states $\sigma, \eta \in\{-1,1\}^{S}$, the probability transiting from $\sigma$ to $\eta$

$$
p_{\sigma \eta}=\prod_{i=1}^{N} \frac{\exp \left(\beta \eta_{i}\left(\sum_{j=1}^{N} T_{i j} \sigma_{j}-U_{i}\right)\right.}{\exp \left(\beta\left(\sum_{j=1}^{N} T_{i j} \sigma_{j}-U_{i}\right)\right)+\exp \left(-\beta\left(\sum_{j=1}^{N} T_{i j} \sigma_{j}-U_{i}\right)\right)},
$$

where $\beta(>0), T_{i j}$ and $U_{i}(1 \leq i, j \leq N)$ are parameters of the model.

$$
{ }^{1} \text { The function } \operatorname{sign}(x)= \begin{cases}1 & x>0 \\ 0 & x=0 \\ -1 & x<0\end{cases}
$$


B2 (continuous time, synchronous): Consider the continuous-time Markov chain $\left\{\xi_{t}\right.$ : $t \geq 0\}$ on state space $\{-1,1\}^{S}$. Every state waits an exponential time with meantime $\lambda^{-1}$ until choosing the next state by the rule of model $B 1$. So the transition density matrix is

$$
q_{\sigma \eta}=\lambda p_{\sigma \eta}, \forall \sigma, \eta \in\{-1,1\}^{S} .
$$

B3 (discrete time, asynchronous): Denote $\sigma^{i}$ to be the new state which changes the sign of the $i$ th coordinate of $\sigma$. Consider the Markov chain $\left\{X_{n}=\left(X_{n}^{1}, X_{n}^{2}, \cdots, X_{n}^{N}\right), n=\right.$ $0,1,2, \cdots\}$ on state space $\{-1,1\}^{S}$. The neurons are updated one by one, in some prescribed sequence, or in a random order. Then choose the next state according to the probability:

$$
p_{\sigma \sigma^{i}}=\frac{\exp \left(-\beta \sigma_{i}\left(\sum_{j=1}^{N} T_{i j} \sigma_{j}-U_{i}\right)\right)}{\exp \left(\beta\left(\sum_{j=1}^{N} T_{i j} \sigma_{j}-U_{i}\right)\right)+\exp \left(-\beta\left(\sum_{j=1}^{N} T_{i j} \sigma_{j}-U_{i}\right)\right)}, \sigma \in\{-1,1\}^{S},
$$

where $\beta>0$; and $p_{\sigma \eta}=0$, if $\eta \neq \sigma^{i}$ for each $i$.

B4 (continuous time, asynchronous): Every state waits an exponential time with meantime $\lambda^{-1}$ until choosing the next state by the rule of model $B 3$. So the transition density matrix is

$$
q_{\sigma \sigma^{i}}=\lambda p_{\sigma \sigma^{i}}, \forall \sigma \in\{-1,1\}^{S}
$$

The third class given below is a variant of the model $B 1$. It is included here since it is the model used for the probabilistic Boolean network of cell-cycle regulation.

\section{Model C: deformation}

Fix $\alpha>0$. Consider a new Markov chain $\left\{X_{n}=\left(X_{n}^{1}, X_{n}^{2}, \cdots, X_{n}^{N}\right), n=0,1,2, \cdots\right\}$ on the state space $\{-1,1\}^{S}$ taking the model $B 1$ as defined initially, with transition probability given as follows:

$$
P\left(X_{n+1} \mid X_{n}\right)=\prod_{i=1}^{N} P_{i}\left(X_{n+1}^{i} \mid X_{n}\right)
$$

where we define

$$
\begin{aligned}
& P_{i}\left(X_{n+1}^{i} \mid X_{n}\right)= \\
& \begin{cases}\frac{\exp \left(\beta X_{n+1}^{i}\left(\sum_{j=1}^{N} T_{i j} X_{n}^{j}-U_{i}\right)\right)}{\exp \left(\beta\left(\sum_{j=1}^{N} T_{i j} X_{n}^{j}-U_{i}\right)\right)+\exp \left(-\beta\left(\sum_{j=1}^{N} T_{i j} X_{n}^{j}-U_{i}\right)\right)} & \text { if } \sum_{j=1}^{N} T_{i j} X_{n}^{j} \neq U_{i} \\
\frac{1}{1+e^{-\alpha}} & \text { if } \sum_{j=1}^{N} T_{i j} X_{n}^{j}=U_{i} \text { and } X_{n+1}^{i}=X_{n}^{i} \\
\frac{e^{-\alpha}}{1+e^{-\alpha}} & \text { if } \sum_{j=1}^{N} T_{i j} X_{n}^{j}=U_{i} \text { and } X_{n+1}^{i}=1-X_{n}^{i} .\end{cases}
\end{aligned}
$$

Remark II.3 The model $C$ differs from $B 1$ when $\sum_{j=1}^{N} T_{i j} X_{n}^{j}-U_{i}=0$, and the latter is also a special case of the former when $\alpha=0$. 


\section{Mathematical Circulation Theory of Network Nonequi- librium Steady States}

There are many different approaches to the theory of nonequilibrium statistical mechanics in the past [9, 21, 16, mathematical theories of which have emerged in the last two decades, and Jiang et.al. have summarized their results of this theory in a recent monograph [14. The most important concepts in the theory are $(i)$ reversibility of a stationary process that corresponds to thermodynamic equilibrium, and (ii) the circulation in a stationary process which corresponds to NESS. A key result of the theory is the circulation decomposition of NESS.

\section{III.1 Circulation theory of nonequilibrium steady states}

Hill [10] constructed a theoretical framework for discussions of vivid metabolic systems, such as active transport, muscle contractions, etc. The basic method of his framework is diagram calculation for the cycle flux on the metabolic cycles of those systems [10]. He successively found that the result from diagram calculation agrees with the data of the numbers of completing different cycles given by random test (Monte Carlo test), but did not yet prove that the former is just the circulation rate in the sense of trajectory of a corresponding Markov chain. In Chapter 1 and 2 of [14], Markov chains with discrete time and continuous time parameter are used as models of Hill's theory on circulation in biochemical systems. The circulation rate is defined in the sense of trajectories and the expressions of circulation rate are calculated which coincide with Hill's result obtained from diagrams. Hence the authors verify that Hill's cycle flux is equivalent to the circulation rate defined in the sense of trajectories.

Below we only state the circulation theory of discrete-time Markov chains, and refer to 14. Chapter 2] for the quite similar circulation theory of continuous-time Markov chains.

First of all, we state the main results, rewritten in terms of the cycle representation of stationary homogeneous Markov chains ([17], Theorem 1.3.1), which is analogous to the Kirchoff's current law and circuit theory in the networks of master equation systems [30].

Theorem III.1 Given a finite oriented graph $G=(V, E)$ and the weight on every edge $\left\{\omega_{e}>0: e \in E\right\}$. If the weight satisfies the balance equation

$$
\sum_{e^{+}=i} \omega_{e}=\sum_{e^{-}=i} \omega_{e}, \forall i \in V
$$

then there exists a positive function defined on the oriented cycles $\left\{\omega_{c}: c=\left(e_{1}, e_{2}, \cdots, e_{k}\right), k \in\right.$ $\left.Z^{+}\right\}$, such that

$$
\omega_{e}=\sum_{e \in c} \omega_{c}, \forall e \in E
$$


For a finite stationary Markov chain $X$ with finite state space $S=\{1,2, \cdots, N\}$, transition matrix $P=\left\{p_{i j}: i, j \in S\right\}$ and invariant distribution $\bar{\pi}=\left\{\pi_{1}, \pi_{2}, \cdots, \pi_{N}\right\}$, one can take its state space to be the group of vertexes, and $E=\left\{e: e^{+}=i, e^{-}=j, p_{i j}>0\right\}$ with weight $\left\{\omega_{e}=\pi_{i} p_{i j}\right\}$. Then notice that (7) is satisfied, since $\sum_{j} p_{i j}=1$ for each $i$ and

$$
\sum_{j} \pi_{i} p_{i j}=\pi_{i}=\sum_{j} \pi_{j} p_{j i}, \forall i \in V
$$

we can conclude that

Corollary III.2 (cycle decomposition) For an arbitrary finite stationary Markov chain, there exists a positive function defined on the group of oriented circuits $\left\{\omega_{c}: c=\left(i_{1}, i_{2}, \cdots, i_{k}\right), k \in\right.$ $\left.Z^{+}\right\}$such that

$$
\pi_{i} p_{i j}=\sum_{c} \omega_{c} J_{c}(i, j), \forall i, j \in V,
$$

where $J_{c}(i, j)$ is defined to be 1 if the cycle c includes the path $i \rightarrow j$, otherwise 0 .

Definition III.3 $\omega_{c}$ is called the circulation along cycle $c$.

For any $i, j \in S, i \neq j$,

$$
\pi_{i} p_{i j}-\pi_{j} p_{j i}=\sum_{c}\left(w_{c}-w_{c_{-}}\right) J_{c}(i, j)
$$

where $c_{-}$denotes the reversed cycle of $c$. Equation (10) is called the circulation decomposition of the stationary Markov chain $X$.

It can be proved that generally the circulation decomposition is not unique, i.e. it is possible to find another set of cycles $\mathcal{C}$ and weights on these cycles $\left\{w_{c} \mid c \in \mathcal{C}\right\}$ which also fit (10).

However, the most reasonable choice of circulation definition is the one defined in the sense of trajectories form the probabilistic point of view. Along almost every sample path, the Markov chain generates an infinite sequence of cycles, and if we discard every cycle when it is completed and at the meantime record it down, then we can count the number of times that a specific cycle $c$ is formed by time $t$, which we denote by $w_{c, t}(\omega)$.

The following theorem is recapitulated from [14, Theorem 1.3.3].

Theorem III.4 Let $\mathcal{C}_{n}(\omega), n=0,1,2, \cdots$, be the class of all cycles occurring until $n$ along the sample path $\left\{X_{l}(\omega)\right\}$. Then the sequence $\left(\mathcal{C}_{n}(\omega), w_{c, n}(\omega) / n\right)$ of sample weighted cycles associated with the chain $X$ converges almost surely to a class $\left(\mathcal{C}_{\infty}, w_{c}\right)$, that is,

$$
\begin{gathered}
\mathcal{C}_{\infty}=\lim _{n \rightarrow+\infty} \mathcal{C}_{n}(\omega), \text { a.e. } \\
w_{c}=\lim _{n \rightarrow+\infty} \frac{w_{c, n}(\omega)}{n}, \text { a.e. }
\end{gathered}
$$


Furthermore, for any directed cycle $c=\left(i_{1}, i_{2}, \cdots, i_{s}\right) \in \mathcal{C}_{\infty}$, the weight $w_{c}$ is given by

$$
w_{c}=p_{i_{1} i_{2}} p_{i_{2} i_{3}} \cdots p_{i_{s-1} i_{s}} p_{i_{s} i_{1}} \frac{D\left(\left\{i_{1}, i_{2}, \cdots, i_{s}\right\}^{c}\right)}{\sum_{j \in S} D\left(\{j\}^{c}\right)} .
$$

where $D=\left\{d_{i j}\right\}=I-P=\left\{\delta_{i j}-p_{i j}\right\}$ and $D(H)$ denotes the determinant of $D$ with rows and columns indexed in the index set $H$. The function $\delta_{i j}=\left\{\begin{array}{ll}0, & i \neq j ; \\ 1, & i=j,\end{array}\right.$ is the well known Kronecker delta function.

It is important to emphasize that the circulations defined in the above theorem also satisfy the circulation decomposition relation (10).

The above theorem not only rigorously confirms the Hill's theory, but also gives a prior substitute method of the widely used diagrammatic method. The complexity of directed diagrams and cycles increases rapidly with the number of states in the model, while the determinant interpretation is much more systematic and easy to be applied using the mathematics softwares. But it will still cost excessive time to compute these determinants if there are hundreds of states in the model.

Luckily, a Monte Carlo method using the so-called derived chain method [14, Section 1.2] to compute the cycle fluxes has already been developed according to the above theorem, the main idea of which is just simply to discard every cycle when it is completed and at the meantime record it down so as to count the number of times that a specific cycle $c$ is formed by time $t\left(\right.$ i.e. $\left.w_{c, t}(\omega)\right)$. Then when the time $t$ is long enough, one gets the approximated circulation of the cycle $c$ (i.e. $w_{c} \approx \frac{w_{c, t}(\omega)}{t}$ ).

Actually, the Boolean yeast cell-cycle network model discussed in the next section has 2048 states and we find that the Monte Carlo method is much more efficient than the method of determinant interpretation, because the latter is even impossible to be applied to such a large model upon a normal computer.

The relationship between circulation and NESS is as follows, which is recapitulated from [14, Theorem 1.4.8].

Theorem III.5 Suppose that $X$ is an irreducible and positive-recurrent stationary Markov chain with the countable state space $S$, the transition matrix $P=\left(p_{i j}\right)_{i, j \in S}$ and the invariant probability distribution $\Pi=\left(\pi_{i}\right)_{i \in S}$, and let $\left\{w_{c}: c \in \mathcal{C}_{\infty}\right\}$ be the circulation distribution of $X$, then the following statements are equivalent:

(i) The Markov chain $X$ is reversible.

(ii) The Markov chain $X$ is in detailed balance, that is,

$$
\pi_{i} p_{i j}=\pi_{j} p_{j i}, \forall i, j \in S
$$

(iii) The transition probability of $X$ satisfies the Kolmogorov cyclic condition:

$$
p_{i_{1} i_{2}} p_{i_{2} i_{3}} \cdots p_{i_{s-1} i_{s}} p_{i_{s} i_{1}}=p_{i_{1} i_{s}} p_{i_{s} i_{s-1}} \cdots p_{i_{3} i_{2}} p_{i_{2} i_{1}}
$$


for any directed cycle $c=\left(i_{1}, \cdots, i_{s}\right)$.

(iv) The components of the circulation distribution of $X$ satisfy the symmetry condition:

$$
w_{c}=w_{c_{-}}, \forall c \in \mathcal{C}_{\infty}
$$

Consequently, when the system is in a nonequilibrium steady state, there exists at least one cycle, containing at least three states, round which the circulation rates of one direction and its opposite direction are asymmetric (unequal), so as to cause a net circulation on the cycle. In theoretic analysis, if there is a large separation in the magnitude of the circulation, between few dominant, main cycles and the rest, it gives rise to the stochastic synchronization phenomenon and helps to distinguish the most important main biological pathways, which can be observed in experiments.

\section{III.2 Applied to Hopfield network and Boltzmann machine}

The keys to understand synchronization behavior in stochastic models are $(i)$ establishing a correspondence between a stochastic dynamics and its deterministic counterpart; and (ii) identifying the cyclic motion in the stochastic models.

In the framework of the stochastic theory, deterministic models are simply the limits of stochastic processes with vanishing noise. This is best illustrated in the following proposition.

Proposition III.6 With the same initial distribution, when $\beta \rightarrow \infty$, the model $B_{k}$ converges to the model $A_{k}$ in distribution, for $k=1,2,3,4$.

Proof: From

$$
\begin{gathered}
\lim _{\beta \rightarrow \infty} \frac{\exp \left(\beta \eta_{i}\left(\sum_{j=1}^{N} T_{i j} \sigma_{j}-U_{i}\right)\right)}{\exp \left(\beta\left(\sum_{j=1}^{N} T_{i j} \sigma_{j}-U_{i}\right)\right)+\exp \left(-\beta\left(\sum_{j=1}^{N} T_{i j} \sigma_{j}-U_{i}\right)\right)} \\
= \begin{cases}1, & \eta_{i}\left(\sum_{j=1}^{N} T_{i j} \sigma_{j}-U_{i}\right)>0 ; \\
0, & \eta_{i}\left(\sum_{j=1}^{N} T_{i j} \sigma_{j}-U_{i}\right)<0 ; \\
\frac{1}{2}, & \sum_{j=1}^{N} T_{i j} \sigma_{j}=U_{i} .\end{cases}
\end{gathered}
$$

We shall further discuss the necessary condition for stochastic Boolean networks to have cyclic motion. In the theory of neural networks, one of Hopfield's key results [11, 12] is that for symmetric matrix $T_{i j}$, the network has an energy function. In the theory of Markov processes, having a potential function(Kolmogorov cyclic condition in Theorem III.5) is a sufficient and necessary condition for a reversible process. A connection is established in the following theorem.

Theorem III.7 For $k=1,2,3,4$, the Markov chain $\left\{X_{n}\right\}$ in the model $B_{k}$ is reversible if and only if $T_{i j}=T_{j i}, \forall i, j=1,2, \cdots, N$, i.e. the matrix $T$ is symmetric. 
Proof: According to theorem III.5, we can use the Kolmogorov cyclic condition to complete the proof. Since every two states are communicative, one only needs to consider the cycles consist of three different states.

Denote these states by $\alpha=\left(\alpha_{1}, \alpha_{2}, \cdots, \alpha_{N}\right), \sigma=\left(\sigma_{1}, \sigma_{2}, \cdots, \sigma_{N}\right)$, and $\eta=\left(\eta_{1}, \eta_{2}, \cdots, \eta_{N}\right)$. Consider the cycle $\alpha \rightarrow \sigma \rightarrow \eta \rightarrow \alpha$ then

$$
\begin{aligned}
& \frac{p_{\alpha \sigma} p_{\sigma \eta} p_{\eta \alpha}}{p_{\alpha \eta} p_{\eta \sigma} p_{\sigma \alpha}} \\
= & {\left[\prod_{i=1}^{N} \frac{\exp \left(\beta \sigma_{i}\left(\sum_{j=1}^{N} T_{i j} \alpha_{j}-U_{i}\right)\right)}{\exp \left(\beta\left(\sum_{j=1}^{N} T_{i j} \alpha_{j}-U_{i}\right)\right)+\exp \left(-\beta\left(\sum_{j=1}^{N} T_{i j} \alpha_{j}-U_{i}\right)\right)}\right.} \\
& \times \prod_{i=1}^{N} \frac{\exp \left(\beta \eta_{i}\left(\sum_{j=1}^{N} T_{i j} \sigma_{j}-U_{i}\right)\right)}{\exp \left(\beta\left(\sum_{j=1}^{N} T_{i j} \sigma_{j}-U_{i}\right)\right)+\exp \left(-\beta\left(\sum_{j=1}^{N} T_{i j} \sigma_{j}-U_{i}\right)\right)} \\
& \left.\times \prod_{i=1}^{N} \frac{\exp \left(\beta \alpha_{i}\left(\sum_{j=1}^{N} T_{i j} \eta_{j}-U_{i}\right)\right)}{\exp \left(\beta\left(\sum_{j=1}^{N} T_{i j} \eta_{j}-U_{i}\right)\right)+\exp \left(-\beta\left(\sum_{j=1}^{N} T_{i j} \eta_{j}-U_{i}\right)\right)}\right] \\
& \div\left[\prod_{i=1}^{N} \frac{\exp \left(\beta \eta_{i}\left(\sum_{j=1}^{N} T_{i j} \alpha_{j}-U_{i}\right)\right)}{\exp \left(\beta\left(\sum_{j=1}^{N} T_{i j} \alpha_{j}-U_{i}\right)\right)+\exp \left(-\beta\left(\sum_{j=1}^{N} T_{i j} \alpha_{j}-U_{i}\right)\right)}\right. \\
& \times \prod_{i=1}^{N} \frac{\exp \left(\beta \sigma_{i}\left(\sum_{j=1}^{N} T_{i j} \eta_{j}-U_{i}\right)\right)}{\exp \left(\beta\left(\sum_{j=1}^{N} T_{i j} \eta_{j}-U_{i}\right)\right)+\exp \left(-\beta\left(\sum_{j=1}^{N} T_{i j} \eta_{j}-U_{i}\right)\right)} \\
& \left.\times \prod_{i=1}^{N} \frac{\exp \left(\beta \alpha_{i}\left(\sum_{j=1}^{N} T_{i j} \sigma_{j}-U_{i}\right)\right)}{\exp \left(\beta\left(\sum_{j=1}^{N} T_{i j} \sigma_{j}-U_{i}\right)\right)+\exp \left(-\beta\left(\sum_{j=1}^{N} T_{i j} \sigma_{j}-U_{i}\right)\right)}\right] \\
= & \exp \left\{-\beta \sum_{i, j} T_{i j}\left[\left(\alpha_{i} \sigma_{j}+\sigma_{i} \eta_{j}+\eta_{i} \alpha_{j}\right)-\left(\alpha_{j} \sigma_{i}+\sigma_{j} \eta_{i}+\eta_{j} \alpha_{i}\right)\right]\right. \\
= & \exp \left[\begin{array}{l}
-\beta \sum_{i, j}\left(T_{i j}-T_{j i}\right)\left(\alpha_{i} \sigma_{j}+\sigma_{i} \eta_{j}+\eta_{i} \alpha_{j}\right) \\
-
\end{array}\right.
\end{aligned}
$$

necessity: Let $\eta_{k}=-1, \forall k=1,2, \cdots, N, \alpha_{k}=-1, \forall k \neq i, \alpha_{i}=1$, and $\sigma_{k}=-1, \forall k \neq j$, $\sigma_{j}=1$. So $\sum_{k, l}\left(T_{k l}-T_{l k}\right)\left(\alpha_{k} \sigma_{l}+\sigma_{k} \eta_{l}+\eta_{k} \alpha_{l}\right)=2\left(T_{i j}-T_{j i}\right)$, if the Markov chain is reversible then by Theorem III.5(iii) $p_{\alpha \sigma} p_{\sigma \eta} p_{\eta \alpha}=p_{\alpha \eta} p_{\eta \sigma} p_{\sigma \alpha}$, we can conclude that $T_{i j}=T_{j i}, \forall i, j=1,2, \cdots, N$.

sufficiency: If $T_{i j}=T_{j i}, \forall i, j=1,2, \cdots . N$, then from (15) one has $p_{\alpha \sigma} p_{\sigma \eta} p_{\eta \alpha}=p_{\alpha \eta} p_{\eta \sigma} p_{\sigma \alpha}, \forall \alpha, \sigma, \eta$.

From the above two conclusions, it is obvious that

Corollary III.8 When $T_{i j}=T_{j i}, \forall i, j=1,2, \cdots, N$, there doesn't exist any limit cycle consist of more than two states in model $A_{k}, k=1,2,3,4$.

Remark III.9 Part of the proof for the corollary III.8 can be found in the literature, such as [36]. The proof we give here, however, provides a new point of view from that of stochastic convergence, and is complete. 
Lemma III.10 For $k=1,2,3,4$, the Markov chain $\left\{X_{n}\right\}$ in the model $B_{k}$ is reversible when $\beta$ is zero, and the Markov chain $\left\{X_{n}\right\}$ in the model $C$ is reversible when $\beta$ and $\alpha$ are both zero.

Proof: In the case when $\beta$ and $\alpha$ are both zero, then for arbitrary two states $\sigma$ and $\eta$, we have $p_{\sigma \eta}=\frac{1}{2}$. So the Markov chain $\left\{X_{n}\right\}$ in the model $B_{k}, k=1,2,3,4$, and model $C$ satisfies the Kolmogorov cyclic condition.

\section{Synchronized Dynamics in a Stochastic Network of Yeast Cell-Cycle Regulation}

From biochemical perspective, the microscopic variables for a cellular regulatory network are the concentrations, or numbers, of various mRNAs, regulatory proteins, and cofactors. If all the biochemistry were known, then the dynamics of such a network would be represented by a chemical master equation [7, 19. Unfortunately, much of the required information is not available, nor such a "fully-detailed" model will always be useful. Phenomenologically the concentrations of key players of a biochemical regulatory network can often be reduced to two or three states, such as resting state, activated state, inactivated state, etc. [4]. The interactions between these states are usually determined from experimental data.

\section{IV.1 The stochastic network model of Zhang et. al.}

We now turn to the stochastic model of cell-cycle network as developed by Li et.al. [18] and Zhang et.al. [38, 39]. The model in [18] is a deformation of model $A_{1}$ with the $(0,1)$ representation instead of the $(-1,1)$ representation: Denote the state of $n$-th step as $X_{n}=\left(X_{n}^{1}, X_{n}^{2}, \cdots, X_{n}^{N}\right)$, then the dynamic is

$$
X_{n+1}^{i}=\frac{1}{2}\left[\operatorname{sign}\left(\sum_{j=1}^{N} T_{i j} X_{n}^{j}\right)+1\right], \text { if } \sum_{j=1}^{N} T_{i j} X_{n}^{j} \neq 0
$$

while $X_{n+1}^{i}=X_{n}^{i}$ if $\sum_{j=1}^{N} T_{i j} X_{n}^{j}=0$.

The main results of [18] are that the network is both dynamically and structurally stable. The biological steady state, known as the G1 phase of a cell cycle, is a global attractor of the dynamics; the biological pathway, i.e., the returning to G1 phase after perturbation, is in a globally attracting basin. There is no limit cycle in this model.

Zhang et.al. [38] implemented a probabilistic Boolean network for the cell-cycle protein interaction network. They found that both the biological steady state and the biological pathway are well preserved under a wide range of noise level. The model in [38] is model 
$C$, with the trivial difference of taking the $(0,1)$ representation rather than $(-1,1)$ representation.

Notice that, according to Proposition III.6, when $\beta, \alpha \rightarrow \infty$, this model recovers the deterministic model in [18; hence, it is implicit that there is no dominant cycle with significant circulation when $\beta$ and $\alpha$ are large.

There is an important difference between the previous models and the most recently developed one in [39], which will be investigated currently in more detail. The earlier works all treat the cell mass (volume), implicitly through protein Cln3 (node 1), as a parameter of their models rather than a dynamic part of the models. Under this setting, the G1 phase of the cell cycle, $(0,0,0,0,1,0,0,0,1,0,0)$ in our binary representation, is a global attractor. The biological cell cycle, however, has a clear sense of cycling with direction. It is a time-irreversible process accompanied with positive entropy production; it is a system in NESS. The cell mass has been incorporated into the transition dynamics in [39], again implicitly characterized by the $\gamma$ parameter: When the system is in G1 phase, a signal to exit the G1 phase by activating Cln3 (node 1), known as START in cell biology, will come with a probability given in Eq. (17) below. It represents the trigger implicitly associated with cell mass. This is the idea of [39], but the method of attack used there is ad hoc with no complete picture.

In the present paper, we focus on the model in [39], thus, is a deformation of model $C$ : fix $\alpha>0, \gamma>0$, consider the Markov chain $\left\{X_{n}=\left(X_{n}^{1}, X_{n}^{2}, \cdots, X_{n}^{N}\right): n=0,1,2, \cdots\right\}$ on the state space $\{0,1\}^{S}$, whose transition probability is as follows:

$$
P\left(X_{n+1} \mid X_{n}\right)=\prod_{i=1}^{N} P_{i}\left(X_{n+1}^{i} \mid X_{n}\right)
$$

where

$$
P_{i}\left(X_{n+1}^{i} \mid X_{n}\right)=\frac{\exp \left(\beta\left(2 X_{n+1}^{i}-1\right)\left(\sum_{j=1}^{N} T_{i j} X_{n}^{j}\right)\right)}{\exp \left(\beta\left(\sum_{j=1}^{N} T_{i j} X_{n}^{j}\right)\right)+\exp \left(-\beta\left(\sum_{j=1}^{N} T_{i j} X_{n}^{j}\right)\right)},
$$

if $\sum_{j=1}^{N} T_{i j} X_{n}^{j} \neq 0$.

$$
P_{i}\left(X_{n+1}^{i} \mid X_{n}\right)= \begin{cases}\frac{1}{1+e^{-\alpha}}, & X_{n+1}^{i}=X_{n}^{i} \\ \frac{e^{-\alpha}}{1+e^{-\alpha}}, & X_{n+1}^{i}=1-X_{n}^{i},\end{cases}
$$

if $\sum_{j=1}^{N} T_{i j} X_{n}^{j}=0$ and $X_{n} \neq(0,0,0,0,1,0,0,0,1,0,0)$ or $i \geq 2$.

$$
P_{1}\left(X_{n+1} \mid X_{n}\right)= \begin{cases}\frac{1}{1+e^{\gamma}}, & X_{n+1}^{1}=X_{n}^{1}, \\ \frac{e^{\gamma}}{1+e^{\gamma}}, & X_{n+1}^{1}=1-X_{n}^{1},\end{cases}
$$

if $\sum_{j=1}^{N} T_{1 j} X_{n}^{j}=0$ and $X_{n}=(0,0,0,0,1,0,0,0,1,0,0)$.

The work [39] only introduced this model which is more reasonable than that in [38], but the results and conclusions in [39] are very short and shallow. So the aim of the present 
paper is to give more profound and comprehensive insight into their work, applying the mathematical theory of nonequilibrium steady states.

The matrix $T$ in all the models above [18, 38, 39] according to Fig 1 is

$$
T=\left[\begin{array}{lllllllllll}
-0.1 & 0 & 0 & 0 & 0 & 0 & 0 & 0 & 0 & 0 & 0 \\
1 & 0 & 0 & 0 & 0 & 0 & 0 & 0 & 0 & -1 & 0 \\
1 & 0 & 0 & 0 & 0 & 0 & 0 & 0 & 0 & -1 & 0 \\
0 & 0 & 1 & -0.1 & 0 & 0 & 0 & 0 & 0 & 0 & 0 \\
0 & 0 & 0 & -1 & 0 & 0 & 1 & -1 & 0 & -1 & 0 \\
0 & 0 & 0 & 0 & 0 & -0.1 & 1 & 0 & 0 & -1 & 1 \\
0 & 0 & 0 & 0 & -0.1 & 0 & 0 & 0 & 0 & 1 & 1 \\
0 & 1 & 0 & 0 & 0 & 0 & -1 & 0 & -1 & 0 & 0 \\
0 & 0 & 0 & -1 & 0 & 1 & 1 & -1 & 0 & -1 & 0 \\
0 & 0 & 0 & 0 & -0.1 & 0 & -1 & 1 & -1 & 0 & 1 \\
0 & 0 & 0 & 0 & 0 & 0 & 0 & 1 & 0 & 1 & -0.1
\end{array}\right],
$$

where $T_{i j}=1$ for a positive regulation of protein $i$ to protein $j$ and $T_{i j}=-1$ for a negative regulation of protein $i$ to protein $j$. If the protein $i$ has a self-degradation loop, $T_{i i}=-0.1$.

This matrix is not symmetric. Hence, according to theorem III.7 the stochastic dynamics has a NESS with nonzero circulation.

Notice that, when $\beta, \alpha$ and $\gamma \rightarrow \infty$, this model converges to a deterministic model similar to that in [18]: Denote the state of n-th step as $X_{n}=\left(X_{n}^{1}, X_{n}^{2}, \cdots, X_{n}^{N}\right)$, then the dynamic is

$$
X_{n+1}^{i}=\frac{1}{2}\left[\operatorname{sign}\left(\sum_{j=1}^{N} T_{i j} X_{n}^{j}\right)+1\right], \text { if } \sum_{j=1}^{N} T_{i j} X_{n}^{j} \neq 0
$$

while $X_{n+1}^{i}=X_{n}^{i}$ if $\sum_{j=1}^{N} T_{i j} X_{n}^{j}=0$ and $X_{n} \neq(0,0,0,0,1,0,0,0,1,0,0)$ or $i \geq 2$; $X_{n+1}^{1}=1-X_{n}^{1}$ if $\sum_{j=1}^{N} T_{1 j} X_{n}^{j}=0$ and $X_{n}=(0,0,0,0,1,0,0,0,1,0,0)$.

The corresponding deterministic model has a limit cycle consist of 13 state. So there is also a main cycle whose circulation is dominant when $\beta, \alpha$ and $\gamma$ are large, which is described by Tab. 1 .

\section{IV.2 Synchronization and dominant circulation in cell-cycle network}

While Zhang et.al. [38, 39] have introduced the probabilistic Boolean network model of yeast cell cycle and tried to describe its nonequilibrium dynamics, they are not fully aware of the important interplay between the the nonequilibrium circulations of this model and the observed synchronization phenomenon.

Furthermore, from numerical computation of the maximal probability flux on cycles, the previous work [38, 39] seems to suggest that the cycle with positive circulation is 


\begin{tabular}{cccccccccccc} 
Cln3 & MBF & SBF & Cln1,2 & Cdh1 & Swi5 & Cdc20/Cdc14 & Clb5,6 & Sic1 & Clb1,2 & Mcm 1/SFF & Phase \\
\hline$(1$ & 0 & 0 & 0 & 1 & 0 & 0 & 0 & 1 & 0 & $0)$ & Start \\
$(0$ & 1 & 1 & 0 & 1 & 0 & 0 & 0 & 1 & 0 & $0)$ & $G_{1}$ \\
$(0$ & 1 & 1 & 1 & 1 & 0 & 0 & 0 & 1 & 0 & $0)$ & $G_{1}$ \\
$(0$ & 1 & 1 & 1 & 0 & 0 & 0 & 0 & 0 & 0 & $0)$ & $G_{1}$ \\
$(0$ & 1 & 1 & 1 & 0 & 0 & 0 & 1 & 0 & 0 & $0)$ & $S$ \\
$(0$ & 1 & 1 & 1 & 0 & 0 & 0 & 1 & 0 & 1 & $1)$ & $G_{2}$ \\
$(0$ & 0 & 0 & 1 & 0 & 0 & 1 & 1 & 0 & 1 & $1)$ & $M$ \\
$(0$ & 0 & 0 & 0 & 0 & 1 & 1 & 0 & 0 & 1 & $1)$ & $M$ \\
$(0$ & 0 & 0 & 0 & 0 & 1 & 1 & 0 & 1 & 1 & $1)$ & $M$ \\
$(0$ & 0 & 0 & 0 & 0 & 1 & 1 & 0 & 1 & 0 & $1)$ & $M$ \\
$(0$ & 0 & 0 & 0 & 1 & 1 & 1 & 0 & 1 & 0 & $0)$ & $M$ \\
$(0$ & 0 & 0 & 0 & 1 & 1 & 0 & 0 & 1 & 0 & $0)$ & $G_{1}$ \\
$(0$ & 0 & 0 & 0 & 1 & 0 & 0 & 0 & 1 & 0 & $0)$
\end{tabular}

Table 1: The synchronous sequence of 13 states as recorded in Li et al. [18

unique. This is not the case. Equipped with Corollary III.2 on cycle decomposition, we shall show that there are many other cycles with positive circulation in the model, although their probability weights are indeed quite small in contrast to the main cycle. This large separation in the magnitudes of the weights gives rise to the stochastic synchronization. Therefore, this is indeed a characterization of the stochastic synchronized dynamics.

Indeed, from the theory of nonequilibrium circulation of discrete-time homogeneous finite Markov chains introduced in Section III, the net circulation is strictly positive as long as the probability of directed cycle is larger than that of its reversal. This is true for any cycle on which the Kolmogorov cyclic condition breaks down [14, Theorem 1.4.8], e.g., in the model in [39], let states $\mu=(0,0,1,0,1,0,0,0,1,0,0), \sigma=(1,0,0,1,0,0,0,0,0,1,0)$, and $\nu=(0,1,0,0,0,0,1,0,0,0,1)$. Consider the cycle $\mu \rightarrow \sigma \rightarrow \nu \rightarrow \mu$. Then

$$
\frac{p_{\mu \sigma} p_{\sigma \nu} p_{\nu \mu}}{p_{\mu \nu} p_{\nu \sigma} p_{\sigma \mu}}=e^{3 \alpha+10.9 \beta} \neq 1 .
$$

Then the net circulation of this cycle is not zero.

Numerical computations with the famous Gillespie's method [7] are carried out, and the results are given in the following figures. The network with 11 binary nodes has a total of 2048 number of states. Following Amit ([1], pp. 75-79), we present the dynamics of the network in terms of integers $0,1,2, \cdots, 2047$, where the state $\left(s_{1}, s_{2}, \cdots, s_{11}\right)$ corresponds one-to-one to $\sum_{i=1}^{11} s_{i} 2^{i-1}$. This 1 -d system obtained is reversible if and only if the 11-d system is reversible.

Fig. 2 is the basic behavior of a random trajectory. The upper panel shows that there arises the phenomenon of local rapid synchronization like that observed in [13] during a very short time period, when $\beta$ is sufficiently large. The lower panel is a random trajectory over a much longer time.

To further characterize the synchronized dynamics, Fig. 3 shows the Fourier power spectrum of the trajectories in Fig. 2, Using MATLAB, the discrete Fourier transform 

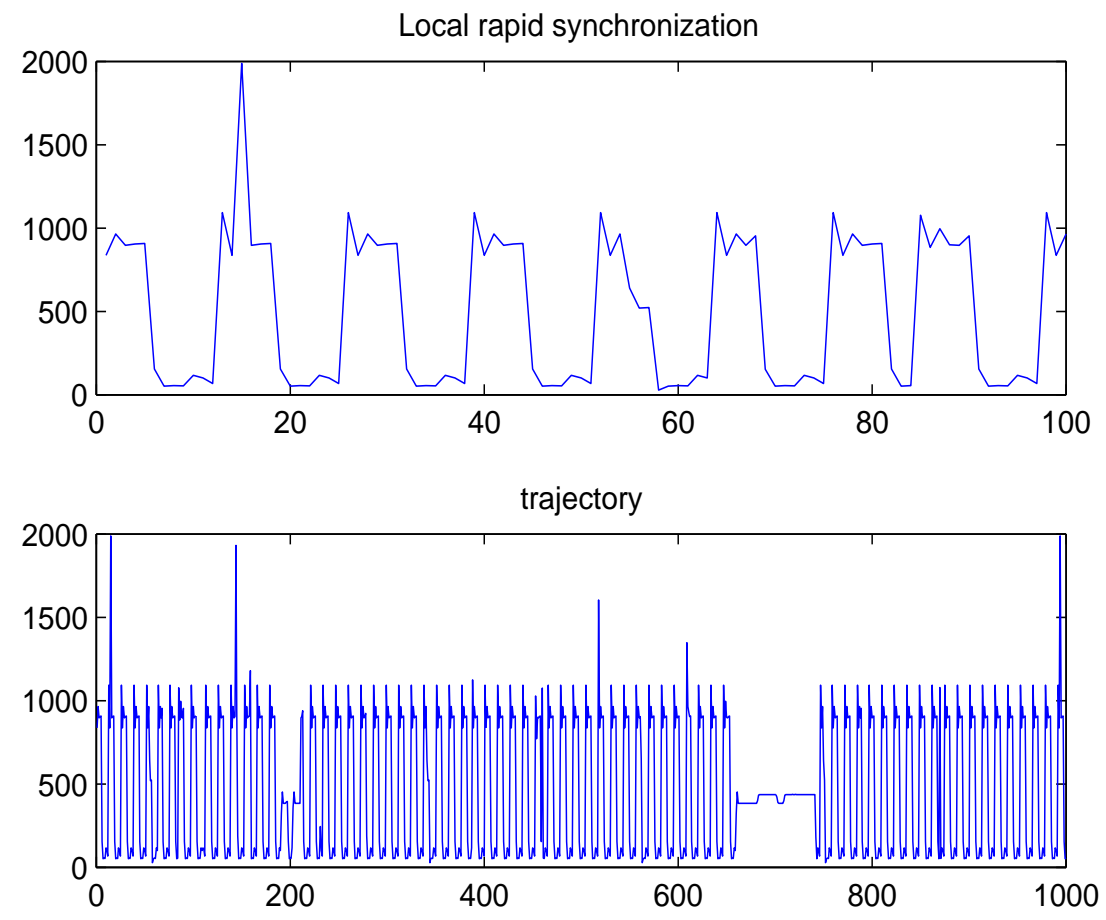

Figure 2: Stochastic trajectory and synchronization. Simulations are carried out with the parameters $\alpha=5, \beta=6$, and $\gamma=5$.

for time series $\left\{x_{1}, x_{2}, \cdots, x_{n}\right\}$ is defined as

$$
y_{m}=\left|\sum_{k=1}^{n} x_{k} e^{-i(2 \pi / n)(m-1)(k-1)}\right|,
$$

which is just the magnitude of frequency $\frac{m-1}{n} 2 \pi, 1 \leq m \leq n$.

Therefore, by the Herglotz theorem ([26], p. 331), the power spectrum of discrete trajectories has a symmetry $y_{m}=y_{n+2-m}$. For different sets of parameters, all the calculations give the distinguishable main peak in the Fig. 3. It is important to mention that different trajectories tend to the same Fig. 3 by ergodicity.

The single dominant peak implies there exists a global synchronization phenomenon. Besides the main peak, the power spectra also have several other smaller peaks, which correspond to several cyclic motions with lower magnitudes. Note that the synchronized behavior is preserved in representation that maps, one-to-one, from the 11 binary nodes to the integers $0-2047$. It is possible that the map will cause some distortion in the power spectrum. To further illustrate the synchronized behavior, Fig. 4 shows the power spectra of all the 11 individual nodes in the network. While subtle details are different, all exhibit the dominant peak, similar to that of the overall dynamics. This demonstrates further the synchronized dynamics in the network.

Fig. 5 plots the magnitude and the period of the dominant peak of the power spectrum 


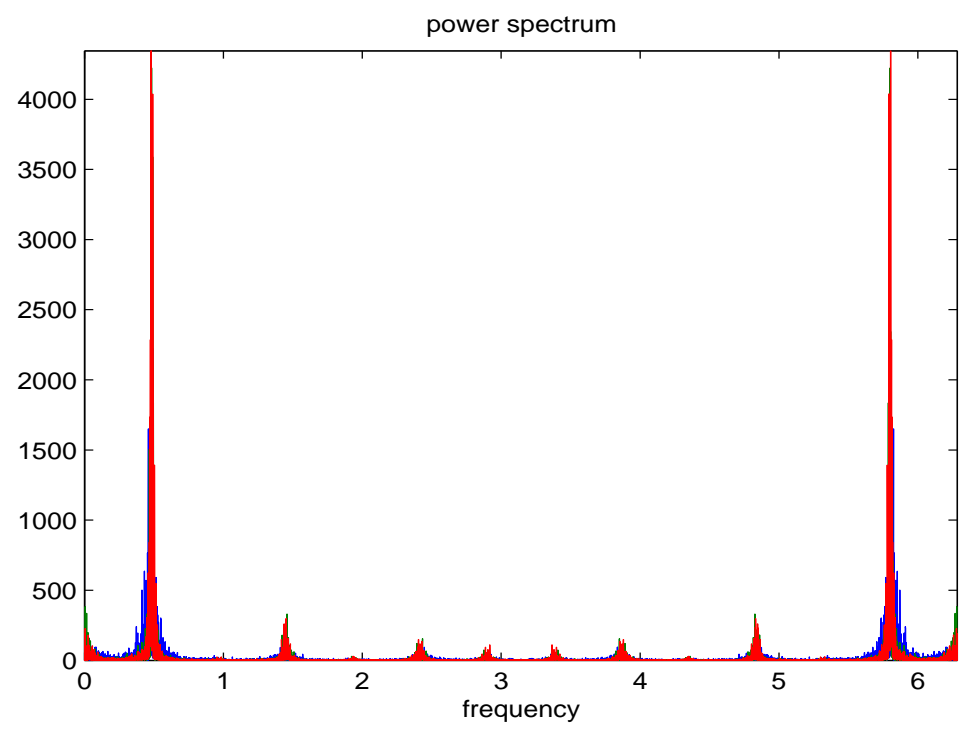

Figure 3: Power spectrum of the overall trajectory with the parameters $\alpha=5, \gamma=5$ fixed, and different $\beta$ : blue: $\beta=2.4$; green: $\beta=4.8$; red: $\beta=6$. The discrete Fourier transform causes an alias; hence the spectrum is symmetric with respect to $\pi$ on the $[0,2 \pi]$ interval.

in Fig. 3, as functions of the noise strength, i.e., the parameter $\beta$. It shows, as we have predicted, that the period converges to 13 which corresponds perfectly to the number of states in the main cycle when $\beta$ is large. We also put error bars on the upper panel of Fig. 5 with various values of $\beta$.

Finally, Fig. [6] shows how the net circulation of the dominant, main cycle varies with $\beta$. It is clearly seen that the net circulation of the main cycle increases monotonically, which implies the appearing of more and more distinct synchronization with increasing $\beta$. The direction of the net circulation does not change.

The net circulations of all the cycles are very small when $\beta, \alpha$ and $\gamma$ are near zero, since the system is close to equilibrium state (reversible) according to lemma III.10.

And for large $\beta$, the net circulations of all the cycles except the main cycle are very small, whose order are about $10^{-6}$ by numerical simulation using the Monte Carlo method for circulation of Markov chain according to Theorem III.4 All the circulations of nonmain cycles actually decrease with increasing $\beta$ when $\beta$ is large.

As in [38, we also notice that there exists an inflection point in the curve in Fig. 6. This implies a cooperative transition of the net circulation of the main cycle while varying the noise level $\beta$. We are currently studying the nature of this phase-transition like behavior. The implication of this observation remains to be further elucidated.

It is also important to point out that the above results are very robust with respect to the values of the matrix $T$ and the threshold $U$, which is similar to the main result in [18, 38. 

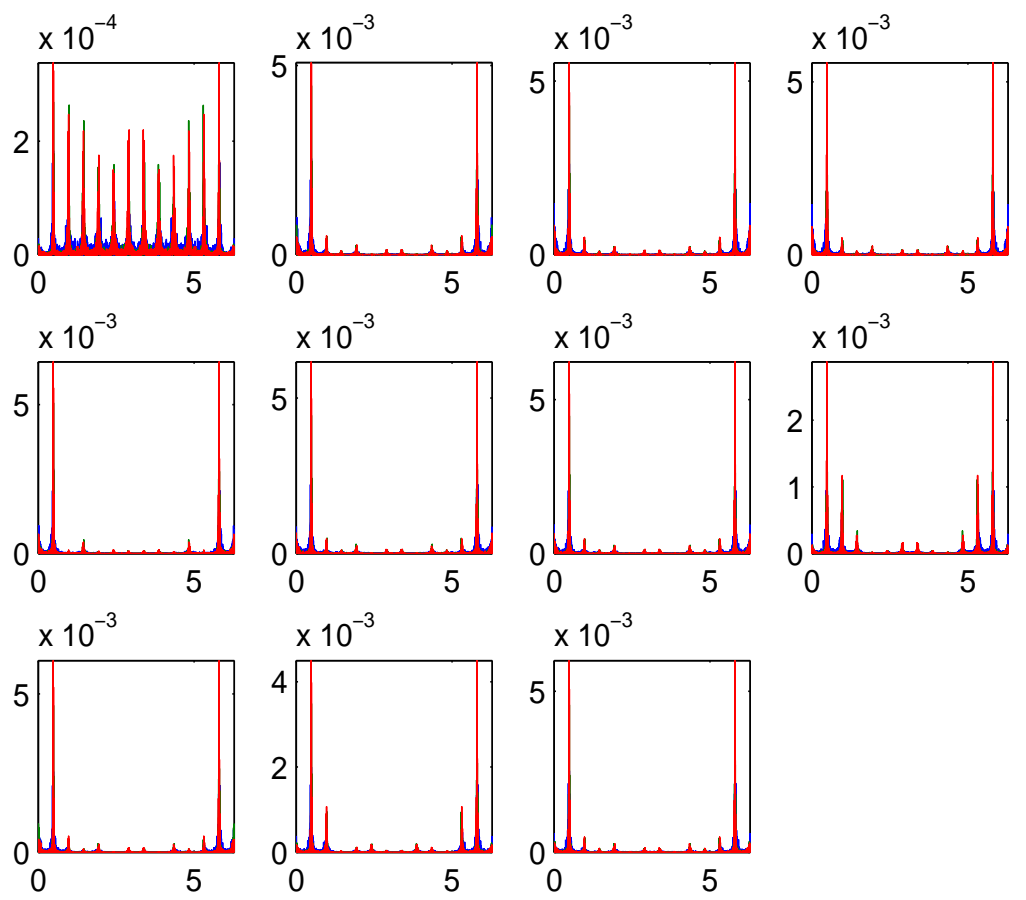

Figure 4: Power spectra of individual nodes show a synchronization among all the nodes with the parameters $\alpha=5, \gamma=5$ fixed, and different $\beta$ : blue: $\beta=2.4$; green: $\beta=4.8$; red: $\beta=6$.

The appearance of the peak in the power spectrum is a signature of a coherent resonance; the existence of circulation means some components of the system are synchronized. A mathematical equivalence between the power spectral peaking and the circulation was proved by Qian et.al. [29] in the case of Markov chains. It has been generalized to all Markov processes by Jiang and Zhang [15].

\section{Conclusion and Discussion}

Quantitative understanding of biological systems and functions from their interacting components presents a significant challenge as well as an unique opportunity for scientists of diverse disciplines. Based on the mathematical theory of nonequilibrium steady states, especially the cycle circulation distribution, synchronized dynamics in a stochastic yeast cell-cycle network is investigated in detail and quantitatively, which is more definite and convincing than the previous method often used by physicists and biologists.

\section{Synchronization and circulation in stochastic network.}

As we know, the occurrence of a deterministic limit cycle in an ordinary differential equation(ODE) model is the hallmark of a synchronization phenomenon. For a stochastic system, such a definite concept no longer holds and a logical generalization needs to 

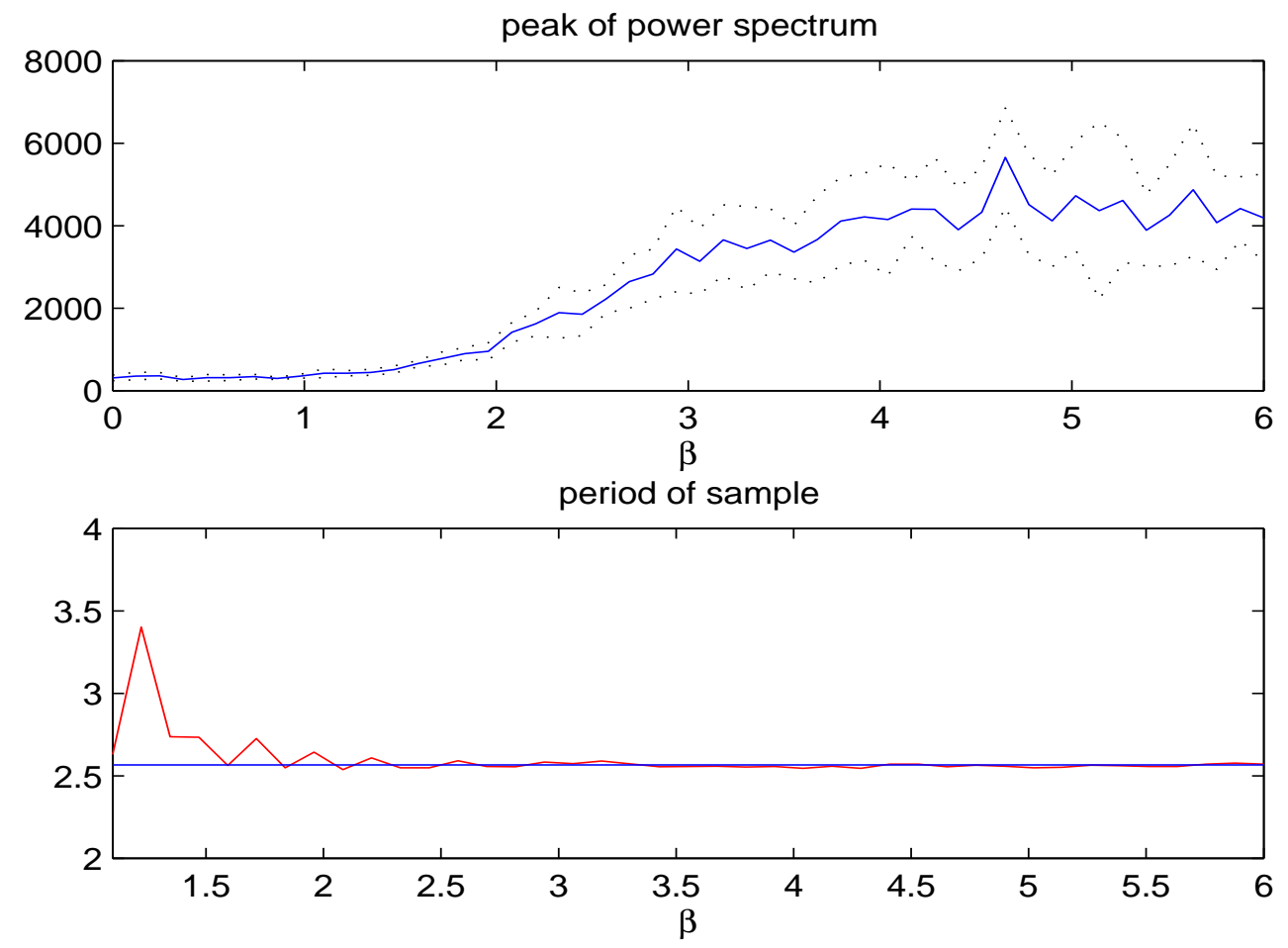

Figure 5: Magnitude and period of the dominant power-spectral peak as functions of $\beta$, with $\alpha=5$ and $\gamma=5$. In the upper panel, the magnitude of the dominant power-spectral peak is averaged over 10 simulations. The solid curve is the mean, and the dotted curves are the mean \pm standard deviation. In the lower panel, the abscissa for the period of the cycle, $T$, is in logarithmic scale. The period approaches to 13 (the horizonal line) with increasing $\beta$.

be investigated. In fact, the very meaning of synchronization requires clarification. In this case, the concept of stochastic limit cycle is useful in describing the synchronization phenomenon. For example, in our present model of yeast cell cycle, the trajectory concentrates around a main cycle, which can be defined as a stochastic limit cycle, is in many aspects very similar to the limit cycle of a deterministic model. Although in many situations this concept does not have a clear definition, it has been rigorously discussed in the context of Markov chain under the heading of circulation theory for many years [27, 28]. We recommend the Chapters 1 and 2 of [14] for the systematic treatment of this theory.

It is shown in the present paper that the stochastic synchronized dynamics in the cell-cycle model, first observed in [38, can be nicely characterized in the perspective of the theory of nonequilibrium steady states and Markov chain circulations. The stochastic Markov chain is reversible (equilibrium state) if and only if the net circulation of every cycle vanishes, which is one of the most important theorems in the mathematical theory 


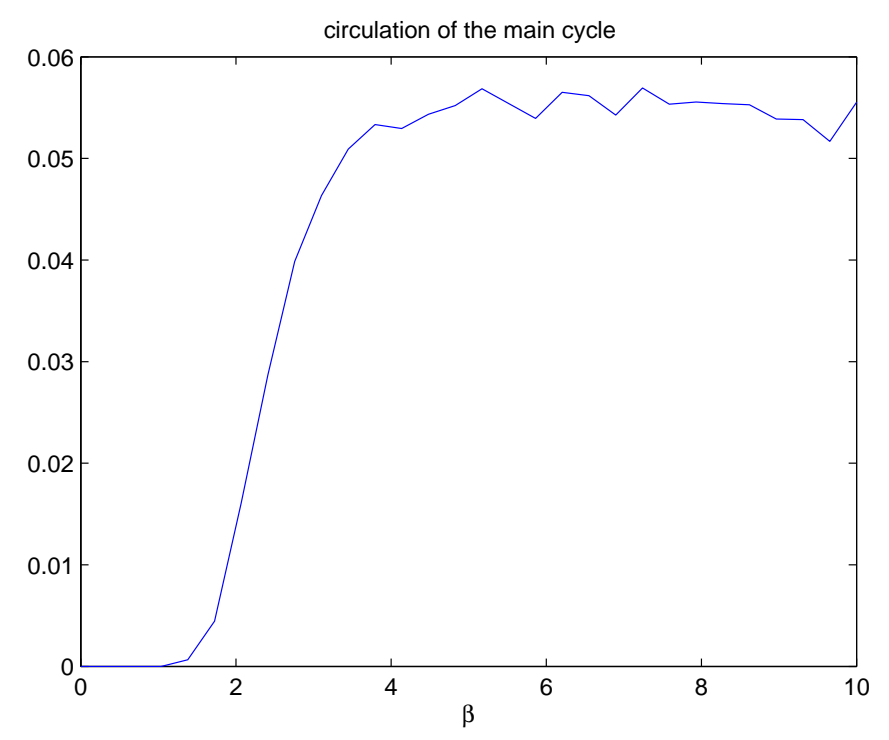

Figure 6: Net circulation of the main cycle as a function of the noise strength, $\beta$.

of NESS.

The stochastic Boolean network is reversible if and only if the matrix $T$ is symmetric, and the net NESS circulation emerges as long as the probability of the directed cycle is larger than that of its reversal, which is just the preconditions for synchronized dynamics to occur. So circulation can be regarded as the quantitative characterization of stochastic synchronized dynamics. Those cycles with high circulation perform stronger synchronized dynamics, while other cycles with low circulation perform weaker synchronized dynamics. Moreover, the distinct global synchronized dynamics can be obviously observed if there is a large scale separation in the magnitude of the circulation between an unique main cycle and the others.

On the other hand, circulation as a good characterization of synchronized dynamics in stochastic models, corresponds perfectly to the power-spectrum algorithm in determining the synchronized dynamics. The magnitudes of the main cycle's circulation and the dominant power-spectral peak both increases monotonically with increasing $\beta$, showing more and more distinct synchronization in the biological systems. Also the period of the dominant power-spectral peak converges to the period(number of states) of the main cycle with dominant circulations.

\section{Stable attractor and robustness in the presence of biological stochasticity.}

Biological systems have to be robust to function in complex (and noisy) environments. More robustness could also mean being more evolvable, and thus more likely to survive. In the model discussed in the present paper, the unique limit cycle in the deterministic network model and the monotonically increasing circulation in the stochastic network model both demonstrate the stability of the main stochastic limit cycle. It can be obviously 
seen that the circulation of the main cycle dominate under a large range of noise level (Fig. 6), which consequently means that this biological function is very robust against small perturbations. It is directly responsible for this cellular process.

Moreover, in some stochastic Boolean network model, one can contain both stable and unstable stochastic limit cycles, where the unstable stochastic limit cycle surprisingly causes vanishing circulation when the noise intensity tends to zero, although it actually is a true limit cycle of the corresponding deterministic Boolean network model which attracts more than half the states in the state space! This phenomenon, called "noise induced global attractive behavior", is recently observed by us in a stochastic Boolean network model of the Siah-1/beta-catenin/p14/19 ARF loop of the protein p53 pathways (unpublished work), in which the biological trajectory, starting from the initial state where only p53 protein is activated, is attracted to the unique stable stochastic limit cycle of the protein states rather than the other unstable one. This fact further confirms the robustness of this biological system. Certainly, more implication of our observations remain to be further elucidated in the future, when applied to other significant bio-problems.

\section{Acknowledgement}

The authors would like to thank Prof. Da-Quan Jiang in Peking University for help suggestion. This work is partly supported by the NSFC(Nos. 10701004, 10531070 and 10625101) and 973 Program 2006CB805900.

\section{References}

[1] Amit, D.J.: Modeling brain function: The world of attractor neural networks. Cambridge University Press 1989

[2] Chen, K.C., Calzone, L., Csikasz-Nagy, A., Cross, F.R., Novak, B., Tyson, J.J.: Integrative analysis of cell cycle control in budding yeast. Mol. Biol. Cell 15 3841-3862 (2004)

[3] Ciliberto, A., Novak, B., Tyson, J.J.: Mathematical model of the morphogenesis checkpoint in budding yeast. J. Cell Biol. 163 1243-1254 (2003)

[4] Fall, C.P., Marland, E.S., Wagner, J.M. and Tyson, J.J.: Computational cell biology. New York: Springer-Verlag 2002

[5] Fox, R.F. and Lu, Y.: Emergent collective behavior in large numbers of globally coupked independently stochastic ion channels. Phys. Rev. E 49(4) 3421-3431 (1994)

[6] Fox, R.F.: Stochastic versions of the Hodgkin-Huxley equations. Biophys. J. 72 2068-2074 (1997) 
[7] Gillespie, D.T.: Exact stochastic simulation of coupled chemical reactions. J. Phys. Chem. 81(25), 2340-2361 (1977)

[8] Goldbeter, A.: Biochemical Oscillations and Cellular Rhythms: the Molecular Bases of Periodic and Chaotic Behaviour, New York: Cambridge Univ. Press 1996

[9] Hasegawa, H.: On the construction of a time-reversed Markoff process, Prog. Theor. Phys. 55, 90-105 (1976); Variational principle for non-equilibrium states and the Onsager-Machlup formula, ibid. 56, 44-60 (1976); Thermodynamic properties of non-equilibrium states subject to Fokker-Planck equations, ibid. 57, 1523-1537 (1977); Variational approach in studies with Fokker-Planck equations, ibid. 58, 128-146 (1977)

[10] Hill, T.L.: Free Energy Transduction and Biochemical Cycle Kinetics. New York: SpringerVerlag 1989

[11] Hopfield, J.J.: Neural networks and physical systems with emergent collective computational abilities. Proc. Natl. Acad. Sci. (79) 2554-2558 (1982)

[12] Hopfield, J.J.: Neurons with graded response have collective computational properties like those of two-state neurons. Proc. Natl. Acad. Sci. (81) 3088-3092 (1984)

[13] Hopfield, J.J., Herz, A.: Rapid local synchronization of action potentials: Toward computation with coupled integrate-and-fire neurons. Proc. Natl. Acad. Sci. (92) 6655-6662 (1995)

[14] Jiang, D.Q., Qian, M. and Qian, M.P.: Mathematical theory of nonequilibrium steady states - On the frontier of probability and dynamical systems. (Lect. Notes Math. 1833) Berlin: Springer-Verlag 2004

[15] Jiang, D.Q. and Zhang, F.X.: The Green-Kubo formula and power spectrum of reversible Markoc processes. J. Math. Phys. 44(10), 4681-4689 (2003)

[16] Keizer, J.: Statistical thermodynamics of nonequilibrium processes. New York: Springer-Verlag 1987

[17] Kalpazidou, S.L.: Cycle representations of Markov processes. (Applications of Mathematics. 28) Berlin: Springer-Verlag 1995

[18] Li, F., Long, T., Lu, Y., et al.: The yeast cell-cycle network is robustly designed. Proc. Natl. Acad. Sci. 101 (14) 4781-4786 (2004)

[19] McQuarrie, D.A.: Stochastic approach to chemical kinetics. J.Appl.Prob. 4(3), 413-478 (1967)

[20] Murray, J.D: Mathematical biology, 3rd Ed. New York: Springer 2002

[21] Nicolis, G. and Prigogine, I.: Self-organization in nonequilibrium systems: from dissipative structures to order through fluctuations. New York: Wiley 1977

[22] Qian, H.: Cycle kinetics, steady-state thermodynamics and motors-a paradigm for living matter physics. J. Phys. Cond. Matt. 17, S3783-S3794 (2005)

[23] Qian, H.: Open-system nonequilibrium steady-state: statistical thermodynamics, fluctuations and chemical oscillations. J. Phys. Chem. B. 110 15063-15074 (2006) 
[24] Qian, H., Saffarian S. and Elson E.L.: Concentration fluctuation in a mesoscopic ocillating chemical reaction system. Proc. Natl. Acad. Sci. USA 99 10376-10381 (2002)

[25] Qian, H. and Reluga, T.C.: Nonequilibrium thermodynamics and nonlinear kinetics in a cellular signaling switch. Phys. Rev. Lett. 94028101 (2005)

[26] Qian, M.P. and Gong, G.L.: Stochastic Processes(second edition). Peking University Press. 1997 (in Chinese)

[27] Qian, M.P. and Qian, M.: Circulation for recurrent Markov chains. Z. Wahrsch. Verw. Gebiete 59, 203-210 (1982)

[28] Qian, M.P., Qian, C. and Qian, M.: Circulations of Markov chains with continuous time and the probability interpretation of some determinants. Sci. Sinica (Series A) 27(5), 470-481 (1984)

[29] Qian, M, Qian, M.P. and Zhang, X.J.: Fundamental facts concerning reversible master equations. Phys. Lett 309, 371-376 (2003)

[30] Schnakenberg, J.: Network theory of microscopic and macroscopic behaviour of master equation systems. Rev. Modern Phys. 48(4), 571-585 (1976)

[31] Scott, A.C.: Neuroscience: A Mathematical Primer. New York: Springer-Verlag 2002.

[32] Strogatz, S.: Sync: The Emerging Science of Spontaneous Order. New York:Hyperion 2003

[33] Van Kampen N.G.: Stochastic Processes in Physics and Chemistry. Amsterdam:NorthHolland 1981

[34] Wilkinson, D.J.: Stochastic Modelling for Systems Biology. Chapman and Hall/CRC 2006

[35] Winfree, A.T.: The Geometry of Biological Time, 2nd. Ed. Berline: Springer-Verlag 2000

[36] Yan, P.F. and Zhang, C.S.: Artificial neural networks and simulating evolutionary computation. Tsinghua University Press 2005 (in Chinese)

[37] Zhou, T.S., Chen, L.N., and Wang, R.Q.: A mechanism of synchronization in interacting multi-cell genetic systems. Physica D 211, 107-127 (2005)

[38] Zhang, Y., Qian, M.P., Ouyang, Q., et al.: Stochastic model of yeast cell-cycle network. Physica D 219, 35-39 (2006)

[39] Zhang, Y., Yu, H., Deng, M.H., Qian, M.P.: Nonequilibrium model for yeast cell cycle. Computational Intelligence and Bioinformatics. International Conference on Intelligent Computing, ICIC2006, Kunming, China, August 16-19, Proceedings, Part III, 786-791 (2006) 